\title{
Susana, narrativa de enfermería y relato hacia el final de su vida: reflexiones desde el pensamiento de Hildegard Peplau
}

\author{
Susana, nurrative of nursing and story to the end of \\ his life: final reflections from the thought of \\ Hildegard Peplau
}

\section{Susana, narrativa da enfermagem e história ao fim de sua vida: reflexões do pensamento do Hildegard Peplau}

Raquel Céspedes Pinto ${ }^{1}$

${ }_{1}$ Enfermera MsC Raquel Céspedes Pinto, Universidad de Santander, facultad Ciencias de la Salud, grupo de Investigación de Enfermería EVEREST. correo electrónicvo: rcespin@yahoo.es.

\section{Cómo citar este artículo en edición digital: Céspedes-Pinto R. (2019). Susana, narrativa de enfermería y relato hacia el final de su vida: reflexiones desde el pensamiento de Hildegard Peplau. Cultura de los Cuidados (Edición digital), 23 (54). Recuperado de http://dx.doi.org/10.14198/cuid.2019.54.08 Correspondencia: Calle 70 N. ${ }^{\circ} 55-210$. Bucaramanga-Santander, Colombia}

Correo electrónico de contacto: rcespin@yahoo.es Recibido: 08/12/2018; Aceptado: 12/03/2019

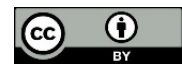

\section{ABSTRACT}

In nursing care, narrative emerges as mediation and a key element in the care process, which also determines an epistemological background, supported by scientific advances in the nursing sciences.

Objective: To analyze the epistemological aspects: meaning, metaparadigm, nursing knowledge patterns and worldviews to a service narrative of a Renal Unit.

Methodology: Hildegard Peplau's thought and the guide for the analysis of nursing situations, it were applied used at the University of Santander-UDES.

Results: The "Situation of Nursing" analyzed is located in the vision of reciprocity. Patterns of nursing knowledge were identified, reflecting predominance of the aesthetic pattern.

Conclusion: In the care of adults with peritoneal dialysis, and with the difficulties in accepting their recent terminal diagnosis, a humanized care is essential.

Keywords: Narrative, nursing, chronic renal insufficiency terminal, care.

\section{RESUMO}

No cuidado de enfermagem, a narrativa surge como mediação e elemento-chave no processo de cuidar, o que também determina um pano de fundo epistemológico, sustentado pelos avanços científicos nas ciências da enfermagem.

Objetivo: Analisar os aspectos 
epistemológicos:

significado, metaparadigma, padrões de conhecimento de enfermagem e visão de mundo para uma narrativa de atendimento de uma Unidade Renal.

Metodologia: Aplicou-se o pensamento de Hildegard Peplau e o guia para a análise das situações de enfermagem, utilizado na Universidade de Santander-UDES.

Resultados: A "Situação da Enfermagem" analisada está localizada na visão de reciprocidade. Os padrões de conhecimento de enfermagem são identificados, refletindo predominância do padrão estético.

Conclusão: No atendimento de adultos com diálise peritoneal e com as dificuldades em aceitar seu diagnóstico terminal recente, é essencial um cuidado humanizado.

Palavras chave: Narrativa, enfermagem, terminal de insuficiência renal crônica, cuidado.

\section{RESUMEN}

En el cuidado de enfermería, surge la narrativa como mediación y elemento clave en el proceso del cuidado, que también determina un trasfondo epistemológico, soportado en los adelantos científicos de las ciencias de enfermería.

Objetivo: Analizar los aspectos epistemológicos: significado, metaparadigma, patrones de conocimiento de enfermería y visiones del mundo a una narrativa de un servicio de una Unidad Renal.

Metodología: Se aplicó el pensamiento de Hildegard Peplau y la guía para el análisis de situaciones de enfermería utilizada en la Universidad de Santander-UDES.

Resultados: La "Situación de Enfermería" analizada se ubica en la visión de reciprocidad. Se identifican los patrones de conocimiento de enfermería, reflejando predominancia del patrón estético.

Conclusión: En la atención del adulto con diálisis peritoneal, y con dificultades en la aceptación de su reciente diagnóstico terminal, es imperante un cuidado humanizado.

Palabras clave: Narrativa, enfermería, insuficiencia renal crónica terminal, cuidado.

\section{INTRODUCCIÓN}

En los profesionales de enfermería, se ha visto el trabajo que han venido desarrollando a nivel investigativo, para brindar en la práctica diaria cuidados de excelente calidad al individuo, su familia y su entorno; lo que constituye una fuerza social importante para liderar cambios esenciales, con gran influencia en el planteamiento de políticas de salud, por ser una de las profesiones más conocedoras de las necesidades en salud, individuales $\mathrm{y}$ colectivas, de la sociedad. Durante el desarrollo de nuestra profesión siempre ha existido la preocupación por tener una visión amplia de diferentes aspectos que de una forma $u$ otra se involucran en el quehacer de enfermería, buscando enriquecer nuestro desempeño $\mathrm{y}$ haciéndonos profesionales más íntegros para desenvolvernos en los diferentes escenarios que ofrece el ejercicio de la enfermería.

Para fortalecer y ampliar los conocimientos sobre modelos conceptuales se realizó una revisión bibliográfica de la teorista Hildegard Peplau, quien ha aportado sus ideas a la práctica diaria con su Teoría Enfermería Ppsicodinamica, la elección de éste modelo deja ver que es una estructura teórica de alto nivel de desarrollo considerándose dentro de las grandes teorías (Morse, Solberg, Neander, Bottorff \& 
Johnson, 1990).

De acuerdo a las visiones de la realidad de enfermería y jerarquía estructural del conocimiento según la narrativa y la relación con la teoría, esta facilita la adquisición de conocimientos que ayudan a perfeccionar las prácticas diarias mediante la descripción y control de los fenómenos, permitiendo que los profesionales logren autonomía en su desempeño. El estudio de la teoría le ayuda a desarrollar habilidades analíticas, estimula el razonamiento, aclara los valores y suposiciones que aplican y determinan los objetivos de la práctica.

Hildegard Peplau, a través de su teoría, hace mención a los conceptos identificados en la situación de enfermería, se basa fundamentalmente en la formación en el campo de la psicología, influenciada por los autores que trabajan teorías de la conducta de Ivan Pavlov, la hipótesis del psicoanálisis sobre el papel de la familia durante los primeros años de vida según Sigmund Freud y las teorías de la motivación humana de Abraham Maslow (Peplau, 1990). En su teoría Peplau describe y analiza cuatro fases complejas en la relación enfermera-paciente: orientación, identificación, aprovechamiento y resolución; y seis funciones de la enfermería psicodinámica: función de persona, recurso, función desconocida, función de liderazgo, función de enseñante, consejera y sustituta.

Para esta teorizante la relación enfermerapaciente, aunque desconocidos en el primer encuentro, ella lo acepta como es, responde a todas sus inquietudes, le da educación, lo invita a participar activamente en el proceso salud-enfermedad para que él, a través de sus vivencias entienda y acepte el porqué de su situación. En su orientación, el individuo tiene una necesidad y busca ayuda profesional, la enfermera le ayuda a reconocer $\mathrm{y}$ entender su necesidad $\mathrm{o}$ problema. Es favorecido por la identificación, a través de la enfermera que asume de facilitadora en la exploración de los sentimientos del paciente para ayudarlo a aceptar su enfermedad. Todo esto logrado a través del aprovechamiento donde el paciente se beneficia de lo que se le ofrece a través de la relación. Permitiendo la resolución de las antiguas metas, las cuales se van dejando de lado en la medida que se van adoptando unas nuevas (Durán de Villalobos, 2001).

En la experiencia psícobiológica, situaciones que impulsaron a dar respuestas, se especifican cuatro prácticas: necesidad, frustración, conflicto y ansiedad. Todas estas experiencias proporcionan energía que se transforma en algún tipo de acción. Si la energía se conduce positivamente aparecen respuestas adaptativas y se madura. Cuando la energía se conduce negativamente aparecen respuestas destructivas que disminuyen la capacidad de percepción y relación. La enfermera observa al paciente y ve cómo va respondiendo a la actuación estableciendo metas u objetivos y lo lleva a cabo (pasando de ser una extraña a conocer al paciente), elaborando un plan de cuidados (proceso): primero observa, en seguida diagnóstica, plantea objetivos, ejecuta acciones y finalmente evalúa. Hildergad Peplau concibe la enfermería como un proceso interpersonal y terapéutico que funciona en términos de cooperación con otros procesos humanos que hacen de la salud una posibilidad para los individuos en las comunidades.

La enfermería tiene dos propósitos, apoyar la sobrevivencia del ser humano y recobrar la salud, además de ayudar al enfermo a comprender el significado de sus problemas de salud y aprender de éstas experiencias. Así se construye un modelo donde la 
enfermera logra acercarse al significado psicológico de los acontecimientos, los sentimientos y los comportamientos, para incorporarlos a las intervenciones de enfermería y enseñarle al paciente la mejor manera de sobrellevar su enfermedad, según Peplau tanto el paciente como la enfermera aprenden del proceso de resolución del problema a partir de su relación. En complemento, concibe que la enfermera no solamente es una intermediaria en la realización de órdenes médicas, también piensa y actúa. En las relaciones que se establecen entre pacienteenfermera sucede lo que la autora denomina "disciplina del proceso", donde la enfermera es capaz de identificar y así mismo satisfacer la ayuda que el paciente necesita con la llamada "respuesta profesional disciplinada" (Travelbee, 1966).

\section{NARRATIVA DE ENFERMERÍA}

Estando a cargo del programa de diálisis peritoneal de una Unidad Renal de Bogotá, conocí a una hermosa joven de 25 años, casada, con 3 hijos (12, 10 y 3 años), sus padres habían fallecido desde que era una niña y demás familiares vivían en una vereda de su ciudad natal. El motivo de su presencia en el servicio fue porque necesitaba tratamiento para una enfermedad cuyo diagnóstico acababa de conocer; Insuficiencia Renal Crónica Terminal y tenía que empezar un programa de diálisis. Al observar su rostro sentía que me hablaban sus gestos; veía facies de preocupación, tristeza, desesperanza, angustia e inseguridad ante su situación; fue una comunicación diferente e impactante por su forma de dialogar con confianza y empatía.

Siendo. ... (...) la Enfermera encargada de orientar a los pacientes debía asesorarlos para que eligieran la mejor opción (ventajas/desventajas) sobre las terapias de reemplazo renal; y que de acuerdo a su decisión vieran una esperanza positiva para su estilo de vida. En éste caso repercutía en una familia muy joven la decisión de una paciente llena de temores de perder a su esposo a quién consideraba el alma de su existencia, manifestación que expresó durante nuestra primera entrevista. Su desesperanza se convirtió para mí en un reto como enfermera, veía que mi labor como cuidadora de su bienestar debía ser la mejor para ayudarla a continuar con su vida, y utilizar todas las estrategias posibles para que ella lograra continuar viviendo y disfrutando del amor de su familia.

Usualmente el tiempo determinado en la institución para dicha labor era corto, pero con ella no era posible terminar con unas cuantas palabras de explicación sobre procedimientos y rutinas, había algo más que hacía correr el tiempo tan lento que ninguna de las dos queríamos que terminara. Así pasamos toda la mañana reconociendo, con profundo detalle, todas sus expectativas e identificando todas las oportunidades que tenía de cuidado para ella, al punto que manifiesta que le gustaría continuar hablando conmigo pues sentía que Yo la comprendía.

$\mathrm{Al}$ escucharle tanto amor hacia su esposo, comprendí que le preocupaba perder su imagen corporal porque ésta era importante para él, le atemorizaba que la abandonara por el cambio, decía: "me preocupa pensar lo que dirá mi esposo al colocarme un catéter en el abdomen", y reiteraba que sin su esposo no podría vivir.

Me sensibilizaba cada vez más el ver su realidad, donde la ciencia y el trabajo interdisciplinar se fusionan para alcanzar el bienestar de todos los pacientes, pero en este caso era una realidad muy difícil por todas 
las complicaciones que el grupo sabía se iban a presentar, realidad llena de percepciones y sentimientos negativos por una vida que se estaba esfumando. Nuestros esfuerzos profesionales se quedaban cortos, no era posible detener el tiempo, mucho menos sus síntomas, ni tampoco sus sentimientos, situación que generaba constantemente mis cuidados que permitían disminuir la forma negativa de ver y sentir su enfermedad.

Me preocupaba mucho su condición, sus hijos, su vida en familia y así se lo manifesté, pero a pesar de mis explicaciones sobre los beneficios y desventajas de las terapias, tomó la decisión por la Hemodiálisis ya que para ella su esposo era más importante que su propia salud. Accediendo a su deseo se programó para implante del catéter para Hemodiálisis explicándole los riesgos que se podían presentar durante la realización del procedimiento. Los meses iniciales de su terapia la reconfortaron tanto que resplandecía su belleza, su cuidado personal impecable y su alegría, tanto para su esposo que la acompañaba en todo momento, como para los pacientes que asistían al mismo procedimiento. Se convirtió en un ejemplo de vida irradiando optimismo para todos los pacientes que acudían a la misma terapia, pero muy pronto vimos como su salud fue empeorando día a día y así mismo su estado emocional. Permanecía llorando todo el tiempo, se notaba ansiosa ya que no podía dormir, no se alimentaba bien, perdió el interés por arreglarse, llegaba a la unidad gritando y diciendo que no quería seguir así. Todo el tiempo decía que la ayudáramos para que su esposo no la abandonara, situación que se convirtió en un reto para mí pues desde el primer momento que la conocí, vi en ella a una joven indefensa y con mucha falta de afecto, llevándome a comprender la importancia de la familia, la salud y el amor.

\section{DEFINICIÓN DE LOS 4 CONCEPTOS DEL METAPARADIGMA EN LA NARRATIVA}

A En la narrativa desde el pensamiento de Peplau, se observa la persona, hubo una etapa de valoración, en la cual se reunió y se interpretó toda la información sobre la persona y su entorno, la situación de salud y la necesidad de cuidado. Se identifica que la paciente tenía 25 años, su ciudad natal era Bogotá, estaba casada, tenía 3 hijos con edades entre 12, 10 y 3 años, sus padres habían fallecido desde que era una niña y sus otros familiares vivían en otra ciudad. Al observar el rostro de Susana, ésta presentaba facies de preocupación, tristeza, ansiedad, angustia e inseguridad ante su situación, ya que se estaba enfrentando a una enfermedad muy difícil de comprender y aceptar, pero a la vez, demostraba, confianza y empatía con la Enfermera.

En cuanto al Entorno en el caso de Susana, en la narrativa, el comportamiento y lenguaje dejaban ver que el nivel académico era bajo, vivía en una casa propia, en un barrio humilde, muy lejos de la unidad renal, no pudo estudiar porque desde muy pequeña tuvo que trabajar para poder subsistir, dependía económicamente de su esposo, porque cuando tuvo los hijos él no la dejó trabajar más. La Salud es la palabra símbolo, dice que es lo que hace avanzar de la personalidad hacia una vida creativa, productiva, constructiva y madura. En la narrativa la enfermera estuvo muy receptiva para detectar las necesidades de Susana y especialmente, cuál era la ayuda que requería. La forma en que la enfermera sintió la acción de ayudar a Susana, enmarcó una diferencia en el resultado de la asistencia, desde el punto de vista de 
expansión de la personalidad. Susana, se adaptó a la situación y aprendió algo, como resultado de la experiencia de la enfermedad y de la enfermera. La Enfermera: Se enmarca en una relación entre un individuo que está enfermo o que tiene alguna necesidad y una profesional que se encuentra preparada para reconocer y satisfacer la necesidad de ayuda de ese individuo. La Enfermera logra sus metas mediante la estimulación de las habilidades que tiene el paciente para afrontar sus problemas, lo lleva a obtener una adecuada salud, en un proceso mutuo y de colaboración para solucionar las necesidades. Observa al paciente, elabora el plan de cuidados y percibe como responde a la actuación, por lo que establece metas $u$ objetivos, pasando de pasar de ser una extraña a conocer al paciente.

La situación entre la Enfermera y Susana, es un claro ejemplo de esta teoría, pues la Enfermera asume con todos sus presaberes y profesionalismo la situación de Susana, la encamina a afrontar su problemática y al mismo tiempo le ofrece las soluciones inmediatas o herramientas eficaces para su tratamiento, asumiendo todos los roles que para Peplau debe cumplir o tener una enfermera. Desde un comienzo la relación paciente-enfermera se dio, adecuada a los factores que cada una utilizó y expuso., La enfermera estuvo muy receptiva para detectar las necesidades de Susana, especialmente, y cuál era la ayuda que requería.

Esto se evidencia en la paciencia para esperar el momento oportuno para lograr la empatía y entablar la comunicación, en su delicadeza para brindar cuidado, en su capacidad de gestionar para que el equipo multidisciplinario se comprometiera en el cuidado, en su valor para atender un enfermo con alteraciones en su salud mental, en un hospital para enfermedades orgánicas.

\section{VISIONES DE LA REALIDAD DE ENFERMERÍA Y NARRATIVA}

La teoría de Peplau se basa en la Visión de Enfermería integrativa-interactiva de reciprocidad, ya que considera al ser humano como un ser en constante desarrollo, y en un equilibrio inestable que necesita a otro ser humano para poder desarrollarse abarca la naturaleza reciproca de las interacciones enfermera-paciente, interactuante. Ve a las personas como seres holísticos, cuyos componentes son inseparables y solo pueden analizarse en el contexto de la totalidad, no pueden ser reducidos a partes. Los seres humanos son activos y la interacción con el ambiente es recíproca. El cambio es función de múltiples factores antecedentes, es probabilístico, puede ser continuo o solo para la supervivencia. Las personas responden a las situaciones con base en sus experiencias anteriores y en la forma como las interpretan, la realidad es multidimensional, dependiente de un contexto y relativa. Los métodos para estudiarla pueden ser cualitativos o cuantitativos.

Esta visión tiene un enfoque diferente, sus seguidores comprenden que los seres humanos son complejos e irreductibles, en su comportamiento reflejan la interpretación que hacen de una situación en un momento determinado, por lo cual es impredecible, $y$ no se pueden generalizar. El poder es mutuo, porque tanto la persona como el profesional de enfermería participan (Leddy \& Pepper, 1989).

La situación de enfermería sucedida entre Susana y la Enfermera encaja totalmente en la teoría psicodinámica de Hildegard Peplau, se puede ver a través de ella todas 
las etapas y supuestos que surgen de la teoría. Se evidencia, que hubo una etapa de valoración, en donde se reunió y se interpretó toda la información sobre Susana y su entorno, la situación de salud y la necesidad de cuidado, la Enfermera exploró, con profundo detalle, todas sus expectativas e identificó todas las oportunidades que tenía de cuidado para ella. Para poder reunir la información se puede evidenciar que hubo varios momentos para entrevistarse con la paciente, la familia y los otros miembros del equipo de salud, se llevaron a cabo también exploraciones físicas $\mathrm{y}$ emocionales.

Esta situación se enmarca en las relaciones interpersonales y la capacidad de la Enfermera para poder identificar lo que siente la paciente, para poderla ayudar durante toda la etapa difícil de la evolución de su enfermedad. En esta relación la Enfermera supo asumir sus roles como persona, recursiva, líder, educadora, consejera y sustituta, cuando la escuchaba, entendía y brindaba afecto, al punto que Susana manifiesta que le gustaría continuar hablando ella pues sentía que la comprendía.

En la etapa de intervención se aplicó el plan de cuidados teniendo en cuenta lo que más afectaba a la paciente y que era lo que más estaba afectando su salud, su estado emocional. La Enfermera comprendió la preocupación por su imagen corporal, por el gran amor hacia su esposo y su temor al abandono. Según H. Peplau, las personas viven cuatro tipos de experiencias psicobiológicas, que son: necesidad de cuidado, frustración, conflicto y ansiedad estas experiencias generan energías que se transforman en acciones; en ésta situación vemos claramente reflejadas estas experiencias desde el comienzo de su enfermedad hasta el momento de su muerte.
En cuanto al cuidado la Enfermera se propuso unas metas: lograr que la paciente aceptara que su vida y bienestar eran más importantes que cualquier persona o cosa y convencer a la paciente para que aceptara su estado de salud y por consiguiente el tratamiento adecuado para su bienestar.

\section{PATRONES DE CONOCIMIENTO}

Patrón empírico: se presenta cuando la Enfermera reconoce en la sintomatología de Susana el temor, la angustia y la ansiedad que vive en ese momento y que la llevan a tomar la decisión de aceptar el tratamiento respectivo, con la clara convicción que puede manejar el cuidado apoyada en un equipo multidisciplinario y que puede brindar cuidado directamente, basándose en sus propios conocimientos empíricos, los mismos que le otorgan la capacidad para explicarle lo que le está ocurriendo, y le permiten crear estrategias de intervención a largo plazo. Adicionalmente, cuando antepone un cuidado más humano y se encarga, a pesar del estado anímico de Susana, de entablar una comunicación efectiva, que le revele el significado que para Susana tiene las condiciones que vive, la Enfermera también obedece a este patrón.

El patrón estético se refleja en la situación cuando Susana, es tratada con dignidad, amablemente, con la calidad y calidez que merece un ser humano, cuando hay una mirada directa y un cuidado humanizado; en la curación a tiempo, percibida como derecho de Susana y no como obligación profesional, transmitiéndole optimismo, esperanza y deseos de vivir, convocando a una acción conjunta para mejorar la calidad de vida. También se manifiesta cuando se interpreta su estado de ánimo psicológico como un llamado angustioso, una necesidad de compañía, amparo y apoyo y no como un 
atentado, y se responde brindando una mano sanadora y compasiva.

El patrón ético le señala el camino que deben recorrer las acciones de la Enfermera. Se muestra a la paciente como una persona con grandes necesidades, que le revela su fragilidad física y emocional, su soledad familiar y social, y lo acepta como su responsabilidad y en consecuencia convoca a todos los miembros del equipo de salud para garantizar condiciones de cuidado que garanticen rehabilitación, toma de decisiones que involucran a todos, y rebasa los límites de la atención conservando únicamente la prudencia. La Enfermera se mostró tal y como es a pesar de la incertidumbre que le generó la situación emocional de la paciente, logrando una empatía que le permitió entablar una relación terapéutica cuando le dio a conocer las ventajas y desventajas que tenía cada terapia para que ella pudiera asumir su enfermedad con claridad.

El patrón de conocimiento personal es su conocimiento de sí misma que le permite valorar sus condiciones para garantizar el cuidado, y delimitar sus alcances. Se ejerce cuando la Enfermera acepta hacerse cargo de una persona con un problema renal y adicionalmente con problemas de familia aunque no está en un centro de caridad, lo acoge bajo la determinante de que se le pueden ofrecer expectativas de vida que harán de su enfermedad algo viable para su bienestar, a la vez que se impone el reto de llevarla a superar sus falencias afectivas y de personalidad. Sus cualidades personales la llevan a interactuar positivamente, a no perder la paciencia ante la actitud de negación de la enfermedad, a no intimidarse ante los estados de decaimiento de ánimo, y a crear un ambiente de empatía donde es importante la consideración del actuar propio como si ella fuera el objeto del cuidado y no el cuidador.

\section{DISCUSIÓN DE RESULTADOS}

En el presente artículo, se ha desarrollado el modelo teórico de Hildegard E. Peplau, a través del mismo, deja ver una estructura teórica con un alto nivel de desarrollo, es descriptivo y explicativo. Esta propuesta se adapta a la realidad en todos los campos de la práctica de enfermería y permite analizar e interpretar las realidades con los diferentes constructos de los modelos, dejando ver un cuidado más humanizado donde se permite que los sentimientos tengan un papel protagónico e importante

Marriner \& Alligood (1994), describen que la teoría de enfermería psicodinámica de Hildegard Peplau está ubicada entre las grandes teorías cuyo esquema estructural propone sobresalientes ideas y algo que es verdad o demostrable. Las grandes teorías son estructuras conceptuales, casi abstractas, aunque proponen resultados basados en la utilización y la aplicación del modelo en la práctica de enfermería. Otra de las características de las grandes teorías es la cantidad de conceptos, definiciones y proposiciones generales y abstractas, que proyectan una visión sistemática de los fenómenos estableciendo, entre los conceptos de describir, explicar y controlar, los fenómenos de la disciplina (Alligood \& Marriner, 2011).

La teoría de enfermería de Hildegard Peplau no permite realizar pruebas empíricas, por lo tanto no es recomendable emplearla para predecir acciones de enfermería en situaciones particulares. Menciona varios conceptos, orientación, identificación, aprovechamiento $\mathrm{y}$ resolución, salud, medio ambiente $\mathrm{y}$ persona. 
La asunción del modelo, se centró en el desarrollo de la relación terapéutica como proceso básico y buscó proporcionar una relación que pudiera hacer que la persona permaneciese sana. Realizar esto de una manera eficaz significa que las enfermeras deben aprender a emplear la ansiedad experimentada por un paciente o por un familiar para ayudarles a comprender en qué consisten los problemas y el modo en que estos problemas pueden solucionarse. Utilizando la relación de este modo, colaborando, educando y siendo una terapeuta, la enfermera será capaz de generar empatía con los problemas del paciente. Como consecuencia de todo ello, tanto la enfermera como el paciente aprenden y maduran (Grupo de Cuidado, 1998).

Hildergard Peplau afirma que los pacientes tendrán problemas si estas necesidades no son satisfechas, bien porque la ansiedad sea demasiado elevada, bien porque la tensión produce frustración $\mathrm{y}$ conflicto en el paciente. El modelo de Peplau trata del cuidado de alguien mediante una serie de interacciones, es, por tanto razonable describirlo, como un modelo de desarrollo más que como un modelo de sistemas (Grupo de Cuidado, 1998).

La enfermería basa su práctica profesional en una relación enfermera-paciente en la cual la enfermera es un instrumento terapéutico y las interacciones se efectúan para lograr un objetivo o transacción. El análisis de esta narrativa permite mostrar la utilidad en términos de la estructura y organización en el cuidado. El concepto de comunicación se produce como un proceso recíproco que se da en las situaciones de encuentro o desencuentro por los que se comunican pensamientos, sentimientos $y$ actitudes, y se comparten las subjetividades, lo que permite ver el cuidado como una labor humanitaria que facilita el crecimiento de cada uno en un proceso que difiere en cada caso.

\section{CONCLUSIONES}

Esta narrativa ha ayudado a desarrollar analíticamente las habilidades, cambios de pensamiento, claridad para evaluar y determinar los propósitos de la práctica de la enfermería, la docencia y la investigación. El modelo de la Teórica Hildegard Peplau, aplica grandes valores personales y profesionales que permiten a enfermería formular juicios y ejecutar acciones basadas en sus propias convicciones y vivencias personales. El lenguaje de la teoría es coherente con el quehacer de enfermería. La relación enfermerapaciente, convierte a la enfermera en un referente no sólo para éste y su familia, sino también para todo el equipo de atención, logrando, de una parte un cambio de actitud en el paciente y la cuidadora de referencia, y de otra en la percepción de los profesionales de la salud que le atienden.

\section{BIBLIOGRAFÍA}

Alligood, M.R.; Marriner-Tomey, A. (2011)

Modelos y teorias en enfermería. $\left(7^{a}\right.$ ed $)$. Madrid:

Elsevier Science.

Durán de Villalobos M.M. (2001). Enfermería: desarrollo teórico investigativo, pp. 19-23 Bogotá: Universidad Nacional de Colombia.

Grupo de Cuidado (1998). Dimensiones del cuidado. Bogotá: Universidad Nacional de Colombia.

Leddy, S., \& Pepper, J. M. (1989). Bases

conceptuales de la enfermería profesional. Phyladelphia: Lippincott company.

Marriner-Tomey, A., \& Alligood, M. R. (1994). Modelos y teorias en enfermería. Mosby: Doyma Libros.

Morse, J. M., Solberg, S.M., Neander, W.L., Bottorff, J.L. \& Johnson, J.L. (1990). Concepts of caring and caring as a concept. ANS Advances in Nursin Science, 13(1), 1-14. 


\section{Cultura de los Cuidados}

Peplau, H. E. (1990). Relaciones interpersonales en enfermería: un marco de referencia conceptual para la enfermería psicodinámica. Barcelona:

Ediciones Científicas y Técnicas.

Travelbee J. (1966). Interpersonal aspects of nursing, pp.121 Philadelphia: F.A. Davis Co, 